\title{
Nanoscale Science and Energy Needs
}

For the past three years, the U.S. agencies that play a role in science have been working together to find, as Feynman said, room at the bottom. In FY 2001, these agencies joined together to propose the National Nanotechnology Initiative (NNI) to explore the world of a few thousand to a few million atoms. (For more information see www.nano.gov). Because it is at the nanoscale that collections of atoms or molecules attain the properties of materials, it is hardly surprising that materials science has emerged as the major component of this initiative.

The NNI is timely, because we now have the tools for research at the nanoscale. A host of new microscopies have been invented since the discovery of the astounding scanning tunneling microscope. These microscopes not only probe but also manipulate single atoms. Powerful machines have been built to provide very high intensity $x$-rays with which the structure of nanosized molecules can be determined such as proteins and with which nanoscale structures can be characterized in polycrystalline solids. New terascale computers now provide the means to calculate properties of nanoscale assemblies of atoms.

The technology that will emerge from nanoscale science promises to be even more fantastic than that which emerged from microscale science. Microscale science put on our laps stores of knowledge such as existed in only a few of the great libraries of the world and the ability to compute in ways not even the wealthiest industries could do 50 years ago. Nanoscale science takes us to the ultimate in miniaturization and forecasts an even more astounding technology revolution. A strand of DNA contains so much information that even our microscale devices strain at dealing with it, yet nature a few billion years ago discovered how to make nanoscale structures to store and manipulate enough information to create life.

However, before the marvels of nanotechnology are realized, we must understand physics and chemistry at this scale, and we must learn how to manipulate nanoscale objects to make materials and functional devices. The interagency NNI is a bold plan to emphasize nanoscale science, engineering, and technology. This plan includes a strong fundamental research program that is investigator-driven, fundamental research in "grand challenges" facing each of the agencies, centers to bring together researchers from many disciplines, research infrastructure to provide the tools to do nanoscale research, work to understand the societal benefits and perils of this new technology, and finally workforce education and training.

Much of nanoscale science is critical to the principal missions of the Department of Energy (DOE) in science, energy, defense, and environment. For example, nanoscale synthesis and assembly methods will result in significant improvements in solar energy conversion; more energy-efficient lighting; selective catalysts; stronger, lighter materials that will improve efficiency in transportation; highly selective separation membranes; and better sensors and controls to increase efficiency in manufacturing. For these reasons, DOE has been involved in nanoscale science since the early 1980s. Current support for nanoscale science is about $\$ 100$ million, most of it in the Office of Basic Energy Sciences (BES). This total does not include funding for the characterization facilities supported by BES (four synchrotron light sources, three neutron sources, and four electron beam microcharacterization centers), which have a critical role in nanoscale research. We have found that there is much interest in nanoscale problems related to energy. In FY 2001, a request for applications resulted in 745 pre-applications and 417 formal proposals. BES awarded a total of $\$ 16.1$ million to 76 of these applications. The DOE laboratories, which were restricted to four proposals per laboratory, submitted 46 proposals. A total of $\$ 10.4$ million was provided to 12 of these proposals.

Besides the basic research in energyrelated grand challenges, BES is supporting Nanoscale Science Research Centers (NSRCs). The NSRCs are a novel concept that arose from the recognition that the BES scientific user facilities were a critical component in the NNI. The NSRCs are research facilities for synthesis, processing, and fabrication of nanoscale materials. They will be collocated with existing user facilities and other specialized facilities at DOE laboratories, which will provide characterization and analytical capabilities. The NSRCs will be operated in the same way as user facilities but will provide specialized equipment and an interdisciplinary support staff. Access to the centers will be based on peer review. The NSRCs will make it possible to do research requiring specialists in several disciplines and in the use of specialized synthesis, processing, and characterization equipment to be done in one place. Just as the BES user facilities provide resources to use beams of neutrons, electrons, or photons, the NSRCs will provide the intellectual and material resources for multidisciplinary research at the nanoscale level. Based on peer review, proposals for Conceptual Design from Lawrence Berkeley National Laboratory, Oak Ridge National Laboratory, and jointly Sandia National Laboratories and Los Alamos National Laboratory were approved. Engineering design funds have been requested in the fiscal year 2002 budget. Construction could begin in fiscal year 2003.

For the future, we are already looking beyond nano. As we begin to understand nanoscale phenomena, it will be necessary to use nanoscale structures as building blocks and to understand how they interact to form supramolecular complexes and hierarchical assemblies. We want solar cells that capture sunlight and make and store fuels. We want materials with embedded sensors that can respond and warn of imminent failure. We want selfrepairing materials. We want catalysts that will make only what we want and nothing else. Such and more are the challenges of nanoscale research.

More information about nanoscale science in BES can be found at www.sc. doe.gov/production/bes/NNI.htm.

IRAN THOMAS

PATRICIA DEHMER

Iran Thomas is Deputy Associate Director of Science for Basic Energy Sciences in the Department of Energy.

Patricia Dehmer is Associate Director of Science for Basic Energy Sciences in the Department of Energy. 